\title{
1 Dietary patterns at 6, 15 and 24 months of age are 2 associated with IQ at 8 years of age
}

3

4 Lisa G Smithers ${ }^{1}$, Rebecca K Golley ${ }^{2}$, Murthy N Mittinty ${ }^{1}$, Laima Brazionis ${ }^{1}$, Kate

5 Northstone $^{3}$, Pauline Emmett ${ }^{3}$, John W Lynch ${ }^{1,3}$

6

${ }^{1}$ Discipline of Public Health, University of Adelaide, Mail drop DX650550, Adelaide, 5005 Australia ${ }^{2}$ Public Health, Sansom Institute for Health Research, University of South Australia, GPO Box 2471, Adelaide 5001, Australia

${ }^{3}$ School of Social and Community Medicine, University of Bristol, Barley House, Oakfield Grove, Bristol BS8 2BN, UK

\section{CORRESPONDING AUTHOR CONTACT DETAILS:}

Phone: +61883130546

Fax: Not available

Email: lisa.smithers@adelaide.edu.au

\section{ABSTRACT}

Diet supplies the nutrients needed for the development of neural tissues that occurs over the first 2 years of life. Our aim was to examine associations between dietary patterns at 6, 15 and 24 months and intelligence quotient (IQ) scores at 8 years. Participants were enrolled in an observational birth cohort (ALSPAC study, $n=7097$ ). Dietary data was collected by questionnaire and patterns were extracted at each time using principal component analysis. IQ was measured using the Wechsler Intelligence Scale for Children at 8 years. Associations between dietary patterns and IQ were examined in regression analyses adjusted for potential confounding and by propensity score matching, with data imputation for missing values. At all ages, higher scores on a Discretionary pattern (characterized by biscuits, chocolate, sweets, soda, crisps) were associated with 1-2 point lower IQ. A Breastfeeding pattern at 6 months and Home-made contemporary patterns at 15 and 24 months (herbs, legumes, cheese, raw fruit and vegetables) were associated with 1-to-2 point higher IQ. A Home-made traditional pattern (meat, cooked vegetables, desserts) at 6 months was positively associated with higher IQ scores, but there was no association with similar patterns at 15 or 24 months. Negative associations were found with patterns characterized by Ready-prepared baby foods at 6 and 15 months and positive associations with a Ready-to-eat foods pattern at 24 months. Propensity score analyses were consistent with regression analyses. This study suggests that dietary patterns from 6-24 months may have a small but persistent effect on IQ at 8 years.

\section{KEYWORDS:}

Dietary patterns, infant, toddler, intelligence quotient, ALSPAC

\section{ABBREVIATIONS:}

ALSPAC

CSE

FSIQ

HM

IQ
Avon longitudinal study of parents and children

Certificate of secondary education

Full scale intelligence quotient

Home-made

Intelligence quotient 
Principal component analysis

Performance intelligence quotient

Ready-prepared

Verbal intelligence quotient

Wechsler intelligence scale for children

\section{Introduction}

Infant diets supply the nutritional substrate for the rapid growth and brain development that occurs in the first years of life. Evidence from observational studies and randomized trials suggests that the postnatal diet may have a lasting influence on child development, even in the absence of overt nutritional deficiencies. For example, prolonged breastfeeding is thought to lead to a higher intelligence quotient (IQ) scores in childhood [1, 2], while supplementing infants with iron-enriched formula may influence cognitive and behavioral outcomes in childhood [3]. In addition to iron, other nutrients have been linked to child development or cognition $[4,5]$.

Nutrient intakes are correlated and one of the limitations of examining associations between single nutrients and cognitive outcomes is that the synergistic effect of the entire diet is not examined. Although multivariate methods exist to examine whole diets, there is a paucity of studies linking whole diets of infants to cognitive development [6]. One small study $(n=241)$ has shown that a dietary pattern characterised by breastfeeding and consumption of fruits, vegetables and home-made foods at 1 year of age was associated with a $\sim 2.7$ point increase in full scale IQ measured by the Wechsler Preschool and Primary Scale of Intelligence at 4 years of age [7]. Northstone et al showed that dietary patterns at 3 years of age had stronger associations with IQ at 8, than patterns at 4, 7 or 8 years [8], supporting the concept that the cognitive development of younger children may be more sensitive to diet. 
We have previously characterized dietary patterns of 6- and 15-month old children participating in a large population-based birth cohort called the Avon Longitudinal Study of Parents and Children (ALSPAC) [9]. Four patterns were extracted at both ages and three of these patterns were similar. The first pattern common to both ages was characterized by home-cooked meat, vegetables and desserts (henceforth referred to as 'Homemade traditional' pattern). The second pattern was characterized by discretionary foods such as biscuits, sweets and crisps ('Discretionary' pattern), and the third pattern was characterised by and named Ready-prepared baby foods. At 6 and 15 months of age, a fourth pattern was characterised by breastfeeding ('Breastfeeding') and by contemporary-style foods including herbs, legumes, nuts, raw fruit and vegetables (Home-prepared contemporary'), respectively. The aim of this study is to characterize dietary patterns at 24 months of age and examine whether patterns at 6,15 and 24 months are associated with IQ scores at 8 years of age.

\section{Subjects and Methods}

\section{ALSPAC cohort}

ALSPAC is a longitudinal birth cohort study designed to investigate the determinants of health and development [10]. All pregnant women residing in the former county of Avon, UK who were expected to deliver between April 1991 and December 1992 were invited to participate. The core ALSPAC sample consists of 14,541 pregnancies and 13988 infants who survived to 1 year of age. The study sample is broadly representative of the population living in Avon at the time although ethnic minorities, single parents and unmarried couples were slightly underrepresented when compared with the 1991 National Census of women with infants under 1 year residing in Avon. Ethical approval for the study was obtained from the ALSPAC Law and Ethics Committee and local Research Ethics Committees. All participants gave informed consent. 


\section{Dietary data and pattern analysis}

Child food and beverage intake data was collected by caregiver-completed postal

questionnaire when the study child was 6,15 and 24 months of age. To maintain the response rate, postal reminders were sent if questionnaires were not returned within one month.

Caregivers were asked to document the frequency of consumption of foods and beverages commonly fed to infants and toddlers. The questionnaire included 43 food and beverage items at 6 months, 70 items at 15 months and 72 items at 24 months of age. The food items included in the questionnaires were determined by an experienced nutritionist (PE). Questionnaires included specific items on the frequency of consuming foods prepared in the home and ready-prepared retail foods manufactured for infants and toddlers. Participants were asked how old the child was when an item was first introduced and how many times per week they fed their child each food item. Infants who had never been fed an item were given an intake of zero. Only questionnaires with complete information on all diet questions were used for principal component analysis (PCA).

110 PCA analysis of dietary data from the questionnaire at 24 months of age is currently under 111 review (K Northstone personal communication, 2011). However, we conducted a separate 112 PCA analysis to ensure consistency with the procedure we applied at 6 and 15 months.

113 Briefly, non-standardised food and beverage data collected at 24 months of age were entered 114 into PCA analysis with Oblimin rotation. Thirty-one food items with low communalities that 115 made almost no contribution to the PCA solution were removed from the analysis to aid 116 statistical convergence. Four patterns were extracted based on the Scree plot, interpretability 117 and consistency with dietary patterns at 6 and 15 months. Dietary pattern scores were 118 computed by summing the product of a standardized frequency of consumption for each food 119 item by its factor loading. Patterns were approximately normally distributed, had a mean of 
zero and standard deviation of one. Patterns were named based on foods with loadings $\geq|0.3|$.

121 PCA analysis was conducted using PASW software (Version 17.0, Chicago Illinois).

\section{IQ data}

123 At 8 years of age, all ALSPAC children were invited to attend a clinic where their cognitive

124 ability was assessed using an adapted form of the Wechsler Intelligence Scale for Children

125 (WISC) Version III [11]. WISC assessments were administered by trained psychologists. To

126 reduce participant burden, alternate items from each of the 10 WISC subtests was

127 administered (5 verbal and 5 performance subtests), except for the coding subtest which was

128 administered in full. To be comparable with administration of the full WISC assessment, raw

129 scores were calculated by summing the individual items on each subtest and multiplying by 2

130 for picture completion, information, arithmetic, vocabulary, comprehension and picture

131 arrangement, multiplying by 1.67 for similarities and multiplying by 1.5 for object assembly

132 and block design. Raw scores were converted to age-standardized scores and combined to

133 determine Full Scale IQ (FSIQ) and subscales of Verbal (VIQ) and Performance IQ (PIQ).

\section{Covariables}

135 Covariables were decided a priori based on a causal model of influences on infant feeding,

136 nutritional status at birth and IQ. Covariables included; maternal age, education, social class,

137 marital status, tobacco smoking, family income, parity, ethnicity and number of children $(<16$

138 years old) living in the family home. These data were collected by postal questionnaires sent

139 to the mother. Maternal education was reported as the highest completed level on five

140 categories from Certificate of Secondary Education (CSE), Vocational training, O-levels, A-

141 levels and degree or higher. The CSE, O- and A-levels are completed at secondary school. O-

142 levels are usually studied at age 16 and A-levels at 18 years. Social class was categorized

143 according to maternal occupation using standard UK classifications of occupation, ranging

144 from class I (highest), II, III-non-manual, III-manual, IV, and V (lowest) [12]. Marital status 
145 was categorized into the following four categories; first marriage, subsequent marriage/s,

146 formerly married (now divorced, widowed or separated) and never married. Tobacco smoking

147 was divided into three categories; never, quit or smoked in the last 2 months of pregnancy.

148 Family income was divided into five $£ 100$ categories ranging from $£<100$ to $£>400 /$ week.

149 Categories for the number of children living in the family home were none, $1,2,3$ and $\geq 4$.

150 Infant covariables including sex, gestational age at birth, birth weight and singleton/multiple

151 birth information was collected by ALSPAC staff at delivery, from medical records or from

152 birth notification. Stimulation in the home environment was measured by an adaptation of the

153 HOME questionnaire at 18 months of age [13].

\section{Statistical analyses}

155 Linear regression analysis was used to examine associations between dietary pattern scores 156 and FSIQ, subscales of VIQ and PIQ. The first regression model included the four PCA 157 patterns scores extracted at one time point, with subsequent models adjusting for perinatal 158 (sex, gestational age, birth weight, ethnicity, singleton/twin, maternal age and parity) and 159 sociodemographic variables (maternal education, social class, smoking, marital status, family

160 income, HOME score and other children). The final model at 15 and 24 months of age 161 included all covariables and dietary pattern scores at younger ages (i.e. the model at 15

162 months of age included 6 month dietary pattern scores; the model at 24 months of age

163 included 6 and 15 month dietary pattern scores). The beta coefficient in the final model is

164 indicates the independent effect of the current dietary patterns after accounting for past dietary 165 patterns. Regression coefficients $(\beta)$ and $95 \%$ confidence intervals were used to evaluate the 166 strength and precision of the associations between dietary patterns and IQ scores. The primary 167 analysis examined the effect of dietary patterns scores at 6, 15 and 24 months of age on FSIQ, 168 with concurrent adjustment for other patterns, perinatal and sociodemographic variables.

169 Secondary analyses included the effect of dietary patterns on Performance and Verbal 
subscales of IQ, with adjustment for confounders and other dietary patterns. Statistical analyses were conducted using STATA (IC11.0, StataCorp TX, USA).

\section{Multiple Imputation}

173 Multiple imputation was used to address missing data [14]. Imputed datasets were generated

174 using Multiple Imputation by Chained Equations under the missing at random assumption.

175 Missing at random is the probability that a missing value does not depend on itself after

176 accounting for other observed explanatory variables [15]. The imputation model included the

177 four dietary patterns at 6,15 and 24 months of age (i.e. 12 dietary pattern scores in total), all

178 covariables listed above and scores on all three measures of IQ (FSIQ, VIQ, PIQ). Dietary

179 pattern scores and covariables were imputed for those with at least one of the IQ subscales

180 measured at 8 years of age. Twenty imputed datasets were generated, using 50 cycles of

181 regression switching [16]. The distribution of imputed variables was comparable with the

182 non-missing data (data not shown). Regression coefficients for the 20 imputed datasets were

183 combined using Rubin's rules [17]. We report the imputed analyses rather than complete case

184 analyses to address possible non-response bias as some of the potentially confounding

185 variables differed between respondents and non-respondents.

\section{Propensity score}

187 Propensity score matching offers an alternative method for adjusting for confounding [18-20].

188 As a sensitivity analysis, we evaluated the average treatment effect (ATE) of dietary pattern

189 scores on FSIQ, VIQ and PIQ by propensity score matching. Using probit regression, the

190 propensity score was calculated as the probability of being in the highest versus the lowest

191 tertile of dietary pattern scores, conditional on the covariates listed above. Probabilities of

192 being in the highest versus lowest tertile were then matched across the area of common

193 support (where the distribution of propensity scores overlap) using the nearest neighbor

194 matching method with callipers set to $0.2^{*} \mathrm{SD}$ [21]. This method matches participants with 
high and low dietary pattern scores on their probability of being in the highest versus lowest

196 tertile of dietary pattern score, conditional on all covariables. Other methods of propensity

197 score matching showed similar results, such as matching by radius (at 0.01 ) and greedy

198 matching with ratios of high: low pattern scores at 1:2 and 1:5. Propensity scores were

199 matched within each of the imputed dataset, then an overall estimate of ATE and the between-

200 dataset variance was computed using Rubin's rules [17]. Propensity score analyses were

201 conducted using STATA's PSMATCH2 command.

\section{Results}

203 Fig. 1 shows the number of participants at each stage of the study and Table 1 shows the 204 sociodemographic characteristics of ALSPAC participants who had IQ measured at 8 years. 205 Of the eligible cohort $(n=13988)$, dietary pattern analysis was conducted on $7052(50.5 \%)$, $2065610(40.1 \%)$ and $6366(45.5 \%)$ participants at 6,15 and 24 months of age respectively and 207 IQ measurements were conducted on 7097 (50.8\%) participants. Of the participants with IQ measurements, those with complete information for all dietary pattern scores and covariables was $n=1366$. This group differed from participants with one or more data items missing $(\mathrm{n}=5731)$. Those with complete data included higher proportions of children of white

211 ethnicity, mothers of higher social classes, higher educational qualifications and fewer 212 mothers who smoked during pregnancy and were unmarried. Family income and social class comprised the two variables with the biggest proportion of missing socio-demographic data

214 (24.1\% and $20.3 \%$, respectively).

\section{Dietary patterns}

216 Dietary patterns at 6 and 15 months have been described previously [9]. At 24 months of age, 217 four dietary patterns were extracted and are named Home-made traditional, Contemporary, 218 Discretionary and Ready-to-eat foods. Foods with loadings $\geq 0.3$ on the Home-made traditional pattern included potatoes (loading 0.76), other vegetables (0.692), meat products 
221 pattern included legumes (0.592), raw apple (0.488), other raw fruit $(0.557)$, herbs $(0.486)$, cheese (0.461), apple juice (0.394), other fruit juice (0.357) and egg (0.373). Foods loading on the Discretionary pattern included crisps (0.642), sweets (0.597), fizzy drink (0.557), chocolate $(0.544)$, cola $(0.536)$, tomato ketchup (0.488), savoury snacks $(0.414)$, baked beans (0.347) and biscuits (0.304). Finally, food loadings for the Ready-to-eat foods pattern included biscuits (0.650), bread/toast (0.617), breakfast cereal $(0.565)$, yoghurt $(0.333)$, milk pudding (0.330) and cola (-0.304). A complete list of loadings for each dietary pattern and all food items entered into the PCA are provided in Online Resource 1.

\section{Regression analysis: effect of dietary patterns at 6,15 and 24 months of age on} IQ at 8 years

231 Results from imputed analyses are reported because participants with complete data differed

232 from those with missing data on one or more potentially confounding variables. Table 2

233 shows the association between dietary pattern scores at 6 months of age and IQ at age 8 years

234 after adjusting for other pattern scores at 6 months (model 1) and potentially confounding

235 covariables (model 2). Higher scores on the Home-made traditional pattern and the

236 Breastfeeding patterns were associated with higher IQ scores, whereas Ready-prepared baby

237 foods and Discretionary patterns were associated with lower IQ scores at 8 years. Adjustment

238 for potentially confounding variables attenuated the association between dietary patterns and

239 IQ, except for Ready-prepared baby foods pattern, which remained stable. Although

240 confidence intervals are wide, the association between dietary patterns and FSIQ and VIQ

241 was consistent. For example, a one-standard deviation increase in the Discretionary Foods

242 pattern score was associated with approximately 1-point lower FSIQ score ( $\beta$ coefficient -1.15

$243(95 \% \mathrm{CI}-1.80,-0.50)$ as well as lower scores on the Verbal $(-1.23(-1.93,-0.54))$ and

244 Performance $(-0.77(-1.42,-0.12))$ subscales. 
The association between dietary pattern scores at 15 months of age and IQ (Table 3) was

247 consistent with the 6-month data in that patterns were associated with IQ at 8 years (model 1)

248 and effects were attenuated after adjustment for potential confounding (model 2). Because

249 dietary patterns may track over time [22-24], we undertook a third model in which we

250 examined the effect of dietary patterns at 15 months while adjusting for earlier diet at 6

251 months (model 3). In the fully adjusted model, a 1 SD higher score on the Home-made

252 contemporary patterns was positively associated with IQ at age 8 while the Discretionary and

253 Ready-prepared pattern scores were negatively associated, but the effect was somewhat

254 weaker after adjustment. The Home-made traditional pattern at 15 months of age was not an

255 independent predictor of IQ at 8 years after adjustment for potential cofounders and diet at 6

256 months.

258 At 24 months of age, the Contemporary pattern had a positive association and the

259 Discretionary and Home-made traditional patterns had weak negative associations with

260 FSIQ, VIQ and PIQ. The fourth Ready-to-eat foods pattern at 24 months was different from

261 patterns at earlier ages and after adjustment for potential confounding and other dietary

262 patterns, there was some evidence of a positive association with FSIQ $(0.76(0.23,1.29))$ and

263 VIQ scores $(0.90(0.36,1.44))$, but no association with PIQ $(0.37(-0.18,0.92))$. Table 4

264 shows that after adjustment for potentially confounding covariables and for diet at 6 and 15

265 months, all observed effects of diet pattern at 24 months on IQ were less than one IQ point.

266 Propensity score analysis: effect of dietary patterns at 6, 15 and 24 months on 267 IQ at 8 years

268 The direction of the effect in the highest compared with the lowest tertiles of dietary pattern

269 scores (Table 5) was consistent with the findings from the regression analyses. In sensitivity

270 analysis, different ways of categorizing dietary pattern scores to obtain a "high" versus a

271 "low" category of diet for generating the propensity score were explored. Dividing dietary 
pattern scores into tertiles retained the greatest sample while attaining the clearest separation

273 of scores (compared with the highest versus lowest quintiles or dichotomizing pattern scores).

274 Checks of the matching process showed that covariables were well balanced after matching,

275 with standardized differences in covariables between the high versus low tertiles were

276 generally $<10 \%$. The effect size estimated from imputed datasets was more conservative than

277 the complete case analysis. The effect of dietary pattern appears consistently lower on PIQ

278 compared with FSIQ and VIQ. However, the overall effect for dietary pattern scores on IQ

279 was in the range of 1 to 2 IQ points which is similar to the multivariable regression analyses.

280 For example, being in the highest compared with the lowest tertile of Contemporary pattern

281 scores at 24 months of age was associated with an average treatment effect of 2.08 points on

282 FSIQ, given other dietary pattern scores (at 6, 15 and 24 months) and covariables.

283

284

285

286

287

\section{Tertiles of Dietary Pattern scores and Adjustment for Propensity Scores in Regression Analyses}

Effect sizes estimated by regression and propensity scores are not directly comparable. To aid interpretation between these methods of controlling for confounding we conducted regression analyses in the imputed datasets, in which tertiles of dietary pattern scores at 6 months were treated as a continuous diet exposure variables on FSIQ and adjusted for either 1) all other dietary patterns and covariables in their natural units or 2) the propensity score. Tertiles of Home-made traditional pattern scores at 6 months were associated with 0.81 (95\% CI $0.19,1.43)$ and $0.77(0.13,1.41)$ higher FSIQ scores at age 8 years after adjustment for all covariables and for propensity scores, respectively. Associations between tertiles of other pattern scores at 6 months and FSIQ were as follows: Discretionary (adjusted for; all covariables $-1.06(-1.66,-0.45)$, propensity scores $-1.05(-1.69,-0.42))$, Ready-prepared baby foods (all covariables $-0.53(-1.04,-0.03)$, propensity scores $-0.54(-1.09,0.004))$ and Breastfeeding patterns (all covariables $1.12(0.53,1.71)$ propensity scores $1.19(0.57,1.81)$ ). 


\section{Discussion}

298 Our analyses suggest that dietary patterns at 6,15 and 24 months of age make a small contribution to IQ at 8 years of age. The association between dietary patterns and IQ is confounded by sociodemographic factors that differ between participants with complete versus missing data. Therefore multiple imputation was conducted to reduce the risk of nonresponse bias due to missing data, while propensity score matching was performed as a sensitivity analysis to the regressions and to address possible selection bias. The findings from the regression analysis and propensity scores are consistent. A positive association between the Breastfeeding and Contemporary dietary patterns and IQ, and a negative association between the Discretionary dietary pattern and IQ were consistent across age groups and analysis methods. The effect size from each method is similar when compared on similar scales. The propensity scores are calculated using the outermost tertiles of the dietary pattern distribution, the boundaries of which are \pm 2 SD from the mean and therefore the effect size in propensity score-based regressions should be halved when comparing with the effect size in regression analysis. Thus, the effect of a 1 SD higher score in Discretionary pattern at 6 months is associated with lower FSIQ scores of 1.15 (95\%CI 1.8, 0.5, Table 2) in regression and 1.11 (Table 5) in propensity analyses. For illustration, a 1 SD increase in Discretionary scores at 6 months from 1.0 to 2.0 is equivalent to increasing intake of chocolate from $<1$ to 1.5 times/wk, biscuits from 1.5 to 2.5 times/wk and tea from <1 to 1.5 times/wk, although it must be remembered that diet pattern scores reflect simultaneous intake of all foods.

The Discretionary and Home-made traditional patterns were present at all ages suggesting that certain styles of diet emerge and persist throughout the first 2 years. However, associations between patterns and IQ differed over time. In the fully adjusted models, the

321 Discretionary pattern at all ages was negatively associated with IQ, but the Home-made 
traditional pattern at 6 months was positively associated, 15 months was not associated and

32324 months was negatively associated with IQ. The Breastfeeding pattern at 6 months and the

Contemporary patterns at 15 and 24 months appear to reflect healthier-style diets and are favourably associated with IQ. The Ready-prepared baby foods patterns at 6 and 15 months and the Ready-to-eat foods pattern at 24 months are characterised by foods that require little cooking or preparation, but despite this similarity, higher scores on the Ready-made baby foods pattern was associated with poorer IQ but the Ready-to-eat foods pattern was associated with better IQ. This may be because the Ready-to-eat foods pattern is characterised by nutrient-rich foods including bread, breakfast cereal, yoghurt and milk pudding.

The concept that infant diet influences cognitive ability has been demonstrated for breastfeeding [1] but there is only one study examining dietary patterns in infancy and IQ [6]. Gale et al reported that dietary patterns characterized by fruit, vegetables and home-made foods at 6 and 12 months of age were associated with around a 0.18 standardized increase in IQ scores at 4 years ( 2 points), but no association was found with a dietary pattern characterised by breads, savoury snacks, biscuits, pasta and breakfast cereals [7]. Analyses were adjusted for a range of confounders, but the study is limited by the small sample selected from a larger cohort $(n=241 / 1981)$ and complete case analysis. Despite this, effect sizes of our study are consistent with Gale et al. Furthermore, our large sample (n=7097), use of imputation and outcomes based at 8 years provide more convincing evidence of the effect of early life dietary patterns, particularly with respect to effects being sustained into childhood.

Our results suggest that the effect of dietary patterns on FSIQ appear to be driven by VIQ rather than PIQ, which is similar to other studies involving infants [7] and 3-year-old children [8]. It may be argued that that since VIQ is more dependent on knowledge and experience, 
347 the effect of dietary patterns might indicate unmeasured confounding, such as a more

348 nurturing environment or parenting practices. However, it is difficult to explain how the

349 effect of dietary patterns on IQ may differ over time if the effect is solely due to confounding.

350 Whether the effects of dietary patterns on IQ outcomes are due to chance, the nutrient profile

351 of dietary patterns, other factors associated with IQ or limitations of these analyses is not

352 clear.

353

354 One of the ongoing difficulties facing nutritional epidemiologists is how to model diet over

355 time. Repeated measures analysis is designed to model repeated outcomes rather than

356 repeated exposures and a further complexity with paediatric nutrition studies is that the

357 exposure (diet) changes considerably from a milk-based diet at 6 months to family foods at 24

358 months. Thus, it should be noted that a potential limitation of these analyses is that we have

359 not adjusted for the repeated nature of individuals' dietary pattern scores. In the absence of

360 more parsimonious methods for modelling repeated dietary exposures, we adjusted for dietary

361 pattern scores at earlier ages to ascertain the independent contribution of dietary patterns at 15

362 and 24 months. Adjustment for earlier diet did attenuate the effect of current dietary patterns

363 (model 3, Tables 3 and 4). This is supported by other studies suggesting that dietary patterns

364 may track over time [23, 22, 24]. Even with adjustment for previous diet, most current dietary

365 patterns remained associated with IQ and this contributes evidence of a cumulative effect of

366 dietary patterns on IQ.

368 Propensity score analyses are an efficient way of addressing systematic differences in the 369 distribution of covariables between exposed and unexposed participants in observational 370 studies. Differences in covariables between the highest and lowest tertiles of dietary pattern 371 scores were large but became well-balanced after matching, thereby allowing us to compare 
the average treatment effect of diet patterns on IQ in a non-experimental setting. The highest

373 and lowest tertiles of pattern scores resulted in a clear separation in diet exposure while maximising sample size. There is very little literature utilising propensity score methods in paediatric nutrition as we found only 2 studies on breastfeeding and IQ [25, 26], indicating that propensity score matching is underutilised in paediatric nutritional epidemiology.

Despite using sophisticated techniques to account for non-response and selection bias, there is no perfect method for controlling residual and unmeasured confounding in observational studies of complex socially patterned exposures and outcomes like diet and IQ. In regression analyses, the effect of dietary pattern scores on IQ was considerably reduced after adjustment for other covariables, indicating substantial confounding and raising the possibility that residual confounding remains. Imputation of missing data was important because participants with complete data were different from those with incomplete data on many covariables that are associated with dietary patterns in early life (Table 1) [27, 24]. Imputation was conducted on the sample who had IQ measured and it is not possible to know the effect of diet in the cohort who did not have IQ measured. A potential limitation of these analyses is that we did not adjust for effect of current diet at age 8 or for parental IQ (data not available), which is thought to account for a small effect of confounding beyond maternal education [28]. In addition, dietary patterns are likely to be specific to the population from which they were derived and therefore caution is needed in extrapolating these findings to other populations.

The present study provides some of the strongest evidence to date that dietary patterns from 6 to 24 months have a small but robust effect on IQ at 8 years of age, after taking into account a range of potential confounding factors (using traditional regression analysis and propensity score matching) and accounting for bias (using multiple imputation) due to missing data. 
We are extremely grateful to all the families who took part in this study, the midwives for their help in recruiting them, and the whole ALSPAC team, which includes interviewers, computer and laboratory technicians, clerical workers, research scientists, volunteers, managers, receptionists and nurses.

Sources of support: The UK Medical Research Council, the Wellcome Trust (Grant ref: 092731) and the University of Bristol provide core support for the ALSPAC study. JWL is supported by an Australia Fellowship (570120) and RKG with a Postdoctoral Training Fellowship (478115) from the National Health and Medical Research Council of Australia. LGS, LB and MNM are supported by funds from the Australia Fellowship awarded to JWL. KN and PE are partly supported by funding from European Community's Seventh Framework Programme (245012).

LGS, RKG and JWL conceived and designed the project. The ALSPAC investigators, including PE and KN, collected the nutritional data and provided advice on its use. LB conducted PCA analysis at 24 months. LGS analysed the data, with all authors contributing to data interpretation. LGS wrote the first draft of the paper, all authors contributed to further drafts and all have read and approved the final version.

Conflict of Interest: PE and KN have both received support from commercial infant food manufacturers and undertaken invited lectures. The other authors have no conflicts of interest to declare.

\section{REFERENCES}

1. Kramer MS, Aboud F, Mironova E, Vanilovich I, Platt RW, Matush L et al. Breastfeeding and child cognitive development. Arch Gen Psychiatr. 2008;65(5):578-84.

2. Brion M-JA, Lawlor DA, Matijasevich A, Horta B, Anselmi L, Araújo CL et al. What are the causal effects of breastfeeding on IQ, obesity and blood pressure? Evidence from comparing high-income with middle-income cohorts. Int J Epidemiol. 2011. doi:10.1093/ije/dyr020.

3. McCann JC, Ames BN. An overview of evidence for a causal relation between iron deficiency during development and deficits in cognitive or behavioral function. Am J Clin Nutr. 2007;82(4):931-45.

4. Birch E, Garfield S, Hoffman DR, Uauy R, Birch D. A randomized controlled trial of early dietary supply of long-chain polyunsaturated fatty acids and mental development in term infants. Dev Med Child Neurol. 2000;42:174-81.

5. Gordon RC, Rose MC, Skeaff SA, Gray AR, Morgan KM, Ruffman T. Iodine supplementation improves cognition in mildly iodine-deficient children. Am J Clin Nutr. 2009;90(5):1264-71.

6. Smithers LG, Golley R, Brazionis L, Lynch JW. Characterizing whole diets of young children from developed countries and the association between diet and health: a systematic review. Nutr Rev. 2011;69(8):449-67.

7. Gale CR, Martyn CN, Marriott LD, Limond J, Crozier S, Inskip HM et al. Dietary patterns in infancy and cognitive and neuropsychological function in childhood. J Child Psychol Psychiatr. 2009;50(7):816-23.

8. Northstone K, Joinson C, Emmett P, Ness A, Paus T. Are dietary patterns in childhood associated with IQ at 8 years of age? A population-based cohort study. J Epidemiol Community Health. 2011; doi:10.1136/jech.2010.111955. 9. Smithers LG, Brazionis L, Golley RK, Mittinty MN, Northsone K, Emett P, McNaughton SA, Campbell KJ, Lynch JW. Dietary patterns at 6 and 15 months of age and sociodemographic factors. Eur J Clin Nutr. 2012; doi: 10.1038/ejcn.2011.219. 10. Golding J, the ALSPAC Study Team. Children of the Nineties: a resource for assessing the magnitude of long-term effects of prenatal, perinatal and subsequent events. Contemp Rev Obstet Gynaecol. 1996;8:89-92. 
11. Wechsler D. The Wechsler intelligence scale for children. Third edition ed. San Antonio, 446 Texas: The Psychological Coorporation; 1991.

447 12. Office of Population Censuses \& Surveys. Standard occupational classification. London:

448 Her Majesty's Stationery Office; 1991.

449 13. Caldwell BM, Bradley RH. Home observation for measurement of the environment. Little

450 Rock: University of Arkansas; 1979.

451 14. Sterne JA, White IR, Carlin JB, Spratt M, Royston P, Kenward MG et al. Multiple

452 imputation for missing data in epidemiological and clinical research: potential and pitfalls. $\mathrm{Br}$

453 Med J. 2009;29(338):b2393.

454 15. Rubin DB. Inference and missing data. Biometrika. 1976;63(3):581-92. 16. Royston P. Multiple imputation of missing values. STATA Journal. 2004;4(3):227-41. 17. Little RJA, Rubin DB. Bayes and multiple imputation. Statistical analysis with missing data. Second ed. Hoboken, NJ: John Wiley \& Sons; 2002. p. 200-22.

458 18. D'Agostino RB. Propensity score methods for bias reduction in the comparison of a treatment to a non-randomized control group. Stat Med. 1998;17(19):2265-81.

460 19. Rosenbaum PR, Rubin DB. The central role of the propensity score in observational studies for causal effects. Biometrika. 1983;70(1):44-55.

20. Rosenbaum PR, Rubin DB. Constructing a control group using multivariate matched sampling incorporating the propensity score. Am Statist. 1985;39:33-8.

21. Austin PC. Optimal caliper widths for propensity-score matching when estimating differences in means and differences in proportions in observational studies. Pharm Stat. 2010;10:150-61.

22. Northstone K, Emmett P. Are dietary patterns stable throughout early and mid-childhood? A birth cohort study. Br J Nutr. 2008;100:1069-76.

23. Mikkila V, Rasanen L, Raitakari OT, Pietinen P, Viikari J. Consistent dietary patterns identified from childhood to adulthood: The cardiovascular risk in young finns study. Br J Nutr. 2005;93(6):923-31.

24. Robinson S, Marriott L, Poole J, Crozier S, Borland S, Lawrence W et al. Dietary patterns in infancy: the importance of maternal and family influences on feeding practice. Br J Nutr. 2007;98(05):1029-37.

25. Jiang M, Foster EM, Gibson-Davis CM. Breastfeeding and the child cognitive outcomes: a propensity score matching approach. Matern Child Health J. 2010;15(8):1296-307.

26. Iacovou M, Sanz AS. Children's cognitive development: does breastfeeding really make a difference. Longit Life Course Stud. 2010;1(3):89.

27. North K, Emmet P, Avon Longitudinal Study of Pregnancy and Childhood (ALSPAC) Study Team. Multivariate analysis fo diet amonth three-year-old children and associations with socio-demographic characteristics. Eur J Clin Nutr. 2000;54:73-80.

28. Der G, Batty GD, Deary IJ. Effect of breast feeding on intelligence in children: 
Table 1: Characteristics of participants with an IQ measurement at 8 years $(n=7097)$ according to completeness of dietary pattern score and covariable data ${ }^{\mathrm{a}}$

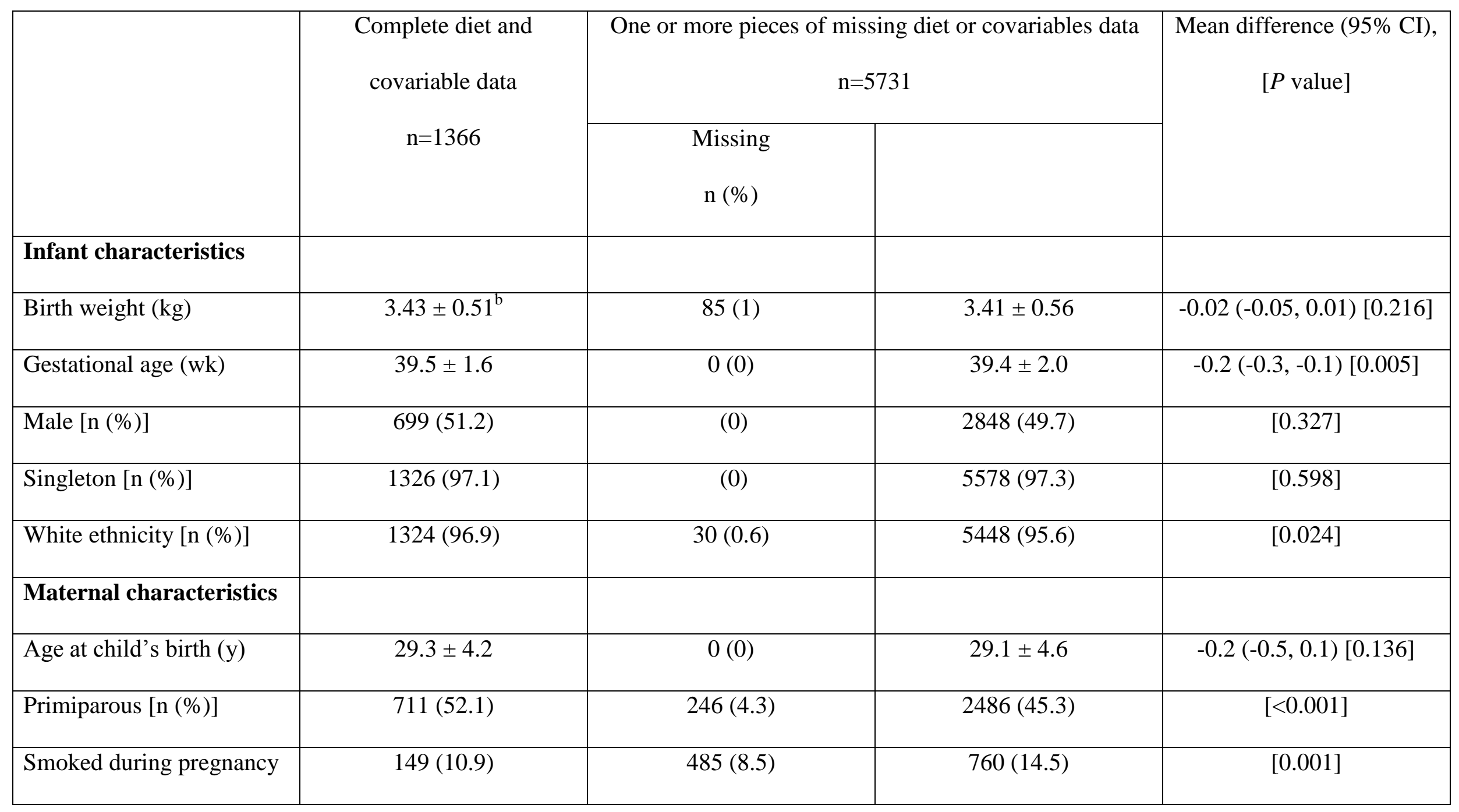




\begin{tabular}{|c|c|c|c|c|}
\hline$[\mathrm{n}(\%)]$ & & & & \\
\hline Social class I or II [n $(\%)]^{\mathrm{c}}$ & $610(44.7)$ & $1166(20.3)$ & $1890(41.4)$ & {$[0.033]$} \\
\hline Family income $>£ 400 /$ wk & $411(30.1)$ & $1382(24.1)$ & $1217(25.9)$ & [0.002] \\
\hline HOME questionnaire $^{\mathrm{d}}$ & $8.2 \pm 2.2$ & $392(6.8)$ & $8.2 \pm 2.2$ & $-0.1(-0.2,0.1)[0.308]$ \\
\hline
\end{tabular}

Abbreviations: PCA, principal component analysis

${ }^{\text {a }}$ Statistical tests were used to compare characteristics of participants who had complete data with those who had one or more pieces of covariable data missing. Continuous variables were compared by using independent $\mathrm{t}$-test and categorical variables were compared by using $\chi^{2}$ test.

${ }^{\mathrm{b}}$ Mean $\pm \mathrm{SD}$ (all such values)

${ }^{\mathrm{c}}$ Social class was categorized according to maternal occupation using UK standard classifications of occupation [12].

${ }^{\mathrm{d}}$ Measured using an adaptation of the HOME observation [13]. 
Table 2: Associations between dietary pattern scores at 6 months of age and full scale, verbal and performance IQ at 8 years of age $(\mathrm{n}=7097)^{\mathrm{a}}$

\begin{tabular}{|c|c|c|c|c|c|c|c|c|c|}
\hline & \multicolumn{3}{|c|}{ Full Scale IQ } & \multicolumn{3}{|c|}{ Verbal IQ } & \multicolumn{3}{|c|}{ Performance $\mathrm{IQ}^{\mathrm{b}}$} \\
\hline & $\beta$ & $95 \% \mathrm{CI}$ & $P$ & $\beta$ & $95 \% \mathrm{CI}$ & $P$ & $\beta$ & $95 \% \mathrm{CI}$ & $P$ \\
\hline \multicolumn{10}{|c|}{ Model 1: adjusted for other dietary pattern at 6 months of age ${ }^{c}$} \\
\hline 6 mo HM traditional & 1.80 & $1.27,2.32$ & $<0.001$ & 1.68 & $1.11,2.26$ & $<0.001$ & 1.48 & $0.99,1.98$ & $<0.001$ \\
\hline 6 mo Discretionary & -3.34 & $-3.98,-2.70$ & $<0.001$ & -3.47 & $-4.15,-2.79$ & $<0.001$ & -2.35 & $-2.98,-1.73$ & $<0.001$ \\
\hline 6 mo RM baby food & -0.65 & $-1.15,0.15$ & 0.011 & -0.86 & $-1.37,-0.35$ & 0.001 & -0.23 & $-0.76,0.31$ & 0.409 \\
\hline 6 mo Breastfeeding & 2.54 & $2.08,3.00$ & $<0.001$ & 2.70 & $2.23,3.18$ & $<0.001$ & 1.69 & $1.19,2.19$ & $<0.001$ \\
\hline \multicolumn{10}{|c|}{ Model 2: adjusted for dietary patterns at 6 months and other covariables ${ }^{c}$} \\
\hline 6 mo HM traditional & 0.69 & $0.18,1.21$ & 0.009 & 0.59 & $0.04,1.15$ & 0.037 & 0.63 & $0.13,1.13$ & 0.014 \\
\hline 6 mo Discretionary & -1.15 & $-1.80,-0.50$ & 0.001 & -1.23 & $-1.93,-0.54$ & 0.001 & -0.77 & $-1.42,-0.12$ & 0.021 \\
\hline 6 mo RM baby food & -0.63 & $-1.06,-0.19$ & 0.005 & -0.84 & $-1.28,-0.39$ & $<0.001$ & -0.20 & $-0.72,0.32$ & 0.442 \\
\hline 6 mo Breastfeeding & 0.97 & $0.49,1.45$ & $<0.001$ & 1.13 & $0.65,1.61$ & $<0.001$ & 0.51 & $0.04,1.06$ & 0.068 \\
\hline
\end{tabular}

${ }^{\mathrm{a}}$ Abbreviations: HM, home-made; RM ready-made; IQ, intelligence quotient.

${ }^{\mathrm{b}}$ IQ was measured by using a short form of the Wechsler Intelligence Scale for Children version III (WISC-III). 
${ }^{c}$ Data were analyzed by multivariate linear regression. In model 1 data were adjusted only for other dietary pattern scores at 6 months. In model

2, data were adjusted for other dietary pattern scores and other potentially confounding variables including: sex, gestational age, birth weight, ethnicity, singleton/twin pregnancies, parity, maternal education, social class, marital status, family income, smoking and HOME questionnaire.

Missing data were imputed by using chained equations. Twenty imputed datasets were created and regression coefficients of each imputed dataset were combined using Rubin's rules [17]. 
Table 3: Associations between dietary pattern scores at 15 months of age and full scale, verbal and performance IQ at 8 years of age $(n=7097)^{a}$

\begin{tabular}{|c|c|c|c|c|c|c|c|c|c|}
\hline & \multicolumn{3}{|c|}{ Full Scale $\mathrm{IQ}^{\mathrm{b}}$} & \multicolumn{3}{|c|}{ Verbal IQ $^{b}$} & \multicolumn{3}{|c|}{ Performance $\mathrm{IQ}^{\mathrm{b}}$} \\
\hline & $\beta$ & $95 \% \mathrm{CI}$ & $P$ & $\beta$ & $95 \% \mathrm{CI}$ & $P$ & $\beta$ & $95 \% \mathrm{CI}$ & $P$ \\
\hline \multicolumn{10}{|c|}{ Model 1: adjusted for other dietary patterns at 15 months of age ${ }^{c}$} \\
\hline 15 mo Contemporary ${ }^{3}$ & 3.36 & $2.88,3.85$ & $<0.001$ & 3.36 & $2.84,3.87$ & $<0.001$ & 2.56 & $2.09,3.03$ & $<0.001$ \\
\hline 15 mo Discretionary & -3.57 & $-4.10,-3.03$ & $<0.001$ & -4.01 & $-4.54,-3.48$ & $<0.001$ & -2.13 & $-2.66,-1.60$ & $<0.001$ \\
\hline 15 mo RM baby food & -1.24 & $-1.93,-0.56$ & 0.001 & -1.27 & $-1.94,-0.60$ & $<0.001$ & -0.88 & $-1.55,-0.20$ & 0.012 \\
\hline 15 mo Rev HM traditional & -0.87 & $-1.43,-0.32$ & 0.003 & -0.82 & $-1.39,-0.26$ & 0.005 & -0.73 & $-1.28,0.18$ & 0.009 \\
\hline \multicolumn{10}{|c|}{ Model 2: adjusted for dietary patterns at 15 months and covariables ${ }^{c}$} \\
\hline 15 mo Contemporary & 1.06 & $0.53,1.59$ & $<0.001$ & 1.07 & $0.50,1.63$ & $<0.001$ & 0.81 & $0.28,1.34$ & 0.003 \\
\hline 15 mo Discretionary & -1.25 & $-1.81,-0.69$ & $<0.001$ & -1.62 & $-2.19,-1.06$ & $<0.001$ & -0.47 & $-1.04,0.10$ & 0.108 \\
\hline 15 mo RM baby food & -1.24 & $-1.84,-0.63$ & $<0.001$ & -1.32 & $-1.92,-0.73$ & $<0.001$ & -0.79 & $-1.41,-0.18$ & 0.012 \\
\hline 15 mo Rev HM traditional & -0.23 & $-0.76,0.30$ & 0.387 & -0.16 & $-0.70,0.38$ & 0.550 & -0.27 & $-0.81,0.27$ & 0.322 \\
\hline \multicolumn{10}{|c|}{ Model 3: model 2 with further adjustments for dietary patterns at 6 months of age } \\
\hline 15 mo Contemporary & 0.67 & $0.07,1.26$ & 0.029 & 0.63 & $0.004,1.25$ & 0.049 & 0.59 & $-0.03,1.21$ & 0.061 \\
\hline
\end{tabular}




\begin{tabular}{|c|c|c|c|c|c|c|c|c|c|}
\hline & \multicolumn{3}{|c|}{ Full Scale IQ ${ }^{b}$} & \multicolumn{3}{|c|}{ Verbal IQ $^{b}$} & \multicolumn{3}{|c|}{ Performance $\mathrm{IQ}^{\mathrm{b}}$} \\
\hline 15 mo RM baby food & -1.11 & $-1.71,-0.50$ & 0.001 & -1.18 & $-1.78,-0.59$ & $<0.001$ & -0.71 & $-1.34,-0.09$ & 0.026 \\
\hline
\end{tabular}

${ }^{a}$ Abbreviations: HM, home-made; mo, months; Rev, reverse; RM, ready-made; IQ, intelligence quotient.

${ }^{\mathrm{b}}$ IQ was measured by a short form of the Wechsler Intelligence Scale for Children version III (WISC-III).

${ }^{\mathrm{c}}$ Data were analyzed by multivariate linear regression. In model 1 data were adjusted only for other dietary pattern scores at 15 months. In model

2, data were adjusted for dietary pattern scores at 15 months and potentially confounding variables including: sex, gestational age, birth weight,

ethnicity, singleton/twin pregnancies, parity, maternal education, social class, marital status, family income, smoking and HOME questionnaire.

Model 3 included all variables described in model 2 as well as adjustment for earlier dietary pattern scores at 6 months of age. Missing data were

imputed by using chained equations. Twenty imputed datasets were created and regression coefficients of each imputed dataset were combined using Rubin's rules [17]. 
Table 4: Associations between dietary pattern scores at 24 months of age and full scale, verbal and performance IQ at 8 years of age $(\mathrm{n}=7097)^{\mathrm{a}}$

\begin{tabular}{|c|c|c|c|c|c|c|c|c|c|}
\hline & \multicolumn{3}{|c|}{ Full Scale $\mathrm{IQ}^{\mathrm{b}}$} & \multicolumn{3}{|c|}{ Verbal IQ $^{\mathrm{b}}$} & \multicolumn{3}{|c|}{ Performance $\mathrm{IQ}^{\mathrm{b}}$} \\
\hline & $\beta$ & $95 \% \mathrm{CI}$ & $P$ & $\beta$ & $95 \% \mathrm{CI}$ & $P$ & $\beta$ & $95 \% \mathrm{CI}$ & $P$ \\
\hline \multicolumn{10}{|c|}{ Model 1: adjusted for other dietary pattern at 24 months of age ${ }^{c}$} \\
\hline 24 mo HM traditional & -0.77 & $-1.23,-0.32$ & 0.001 & -0.79 & $-1.30,-0.29$ & 0.002 & -0.52 & $-1.03,-0.02$ & 0.041 \\
\hline 24 mo Contemporary & 3.01 & $2.55,3.47$ & $<0.001$ & 3.04 & $2.57,3.51$ & $<0.001$ & 2.23 & $1.73,2.74$ & $<0.001$ \\
\hline 24 mo Discretionary & -2.73 & $-3.24,-2.23$ & $<0.001$ & -2.88 & $-3.40,-2.37$ & $<0.001$ & -1.90 & $-2.43,-1.37$ & $<0.001$ \\
\hline 24 mo Ready-to-eat foods & 2.34 & $1.87,2.81$ & $<0.001$ & 2.53 & $2.04,3.01$ & $<0.001$ & 1.94 & $1.02,1.96$ & $<0.001$ \\
\hline \multicolumn{10}{|c|}{ Model 2: adjusted for dietary patterns at 24 months and other covariables } \\
\hline 24 mo HM traditional & -0.37 & $-0.82,0.08$ & 0.104 & -0.37 & $-0.85,0.11$ & 0.133 & -0.24 & $-0.75,0.27$ & 0.349 \\
\hline 24 mo Contemporary & 1.19 & $0.74,1.65$ & $<0.001$ & 1.21 & $0.73,1.69$ & $<0.001$ & 0.88 & $0.34,1.41$ & 0.001 \\
\hline 24 mo Discretionary & -1.12 & $-1.64,-0.61$ & $<0.001$ & -1.24 & $-1.75,-0.74$ & $<0.001$ & -0.73 & $-1.30,-0.16$ & 0.013 \\
\hline 24 mo Ready-to-eat foods & 0.89 & $0.41,1.37$ & $<0.001$ & 1.07 & $0.56,1.57$ & $<0.001$ & 0.42 & $-0.08,0.91$ & 0.099 \\
\hline \multicolumn{10}{|c|}{ Model 3: model 2 with further adjustments for dietary patterns at 6 and 15 months of age } \\
\hline 24 mo HM traditional $^{3}$ & -0.51 & $-1.03,0.01$ & 0.057 & -0.41 & $-0.99,0.17$ & 0.163 & -0.46 & $-1.05,0.13$ & 0.123 \\
\hline
\end{tabular}




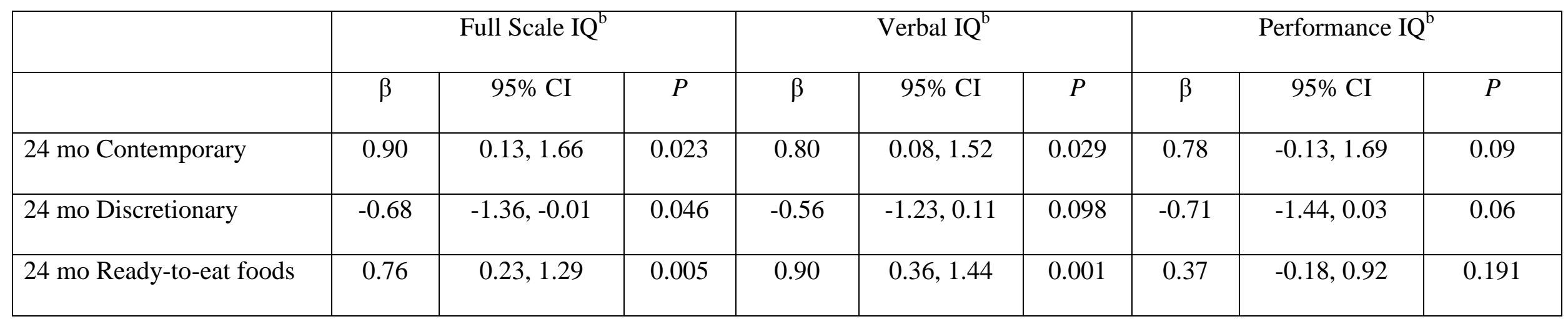

${ }^{a}$ Abbreviations: HM, home-made; mo, months; RM, ready-made; IQ, intelligence quotient.

${ }^{\mathrm{b}}$ IQ was measured by a short form of the Wechsler Intelligence Scale for Children version III (WISC-III).

${ }^{\mathrm{c}}$ Data were analyzed by multivariate linear regression. In model 1 data were adjusted for other dietary pattern scores at 24 months. In model 2 , data were adjusted for dietary pattern scores at 24 months and potentially confounding variables including: sex, gestational age, birth weight, ethnicity, singleton/twin pregnancies, parity, maternal education, social class, marital status, family income, smoking and HOME questionnaire. Model 3 included all variables described in model 2 as well as adjustment for earlier dietary pattern scores at 6 and 15 months of age. Missing data were imputed by using chained equations. Twenty imputed datasets were created and regression coefficients of each imputed dataset were combined using Rubin's rules [17]. 
Table 5: Propensity score analysis: the average treatment effect of being in the highest compared with the lowest tertile of dietary pattern scores at 6 , 15 and 24 months of age on full scale, verbal and performance IQ at 8 years of age ${ }^{\mathrm{a}}$

\begin{tabular}{|c|c|c|c|c|c|c|c|}
\hline & \multicolumn{4}{|c|}{ Average treatment effect by complete case analysis } & \multicolumn{3}{|c|}{ Average treatment effect in imputed datasets ${ }^{b}$} \\
\hline & $\begin{array}{c}\mathrm{n} \text { used in } \\
\text { match }\end{array}$ & FS IQ & V IQ & P IQ & FS IQ & V IQ & P IQ \\
\hline 6 mo HM traditional & 961 & 1.60 & 0.72 & 2.23 & $1.46(0.42)$ & $1.24(0.47)$ & $1.33(0.48)$ \\
\hline 6 mo Discretionary & 889 & -2.50 & -2.21 & -2.27 & $-2.21(0.63)$ & $-2.17(0.69)$ & $-1.68(0.56)$ \\
\hline 6 mo RM baby foods & 1003 & -0.78 & -1.02 & -0.35 & $-1.08(0.37)$ & $-1.60(0.35)$ & $-0.17(0.47)$ \\
\hline 6 mo Breastfeeding & 971 & 3.25 & 3.24 & 2.52 & $2.25(0.47)$ & $2.53(0.50)$ & $1.30(0.48)$ \\
\hline 15 mo Contemporary & 367 & 2.02 & 0.65 & 3.73 & $1.02(0.68)$ & $1.02(0.68)$ & $0.90(0.59)$ \\
\hline 15 mo Discretionary & 432 & 0.22 & -1.37 & 1.96 & $-1.95(0.72)$ & $-2.56(0.84)$ & $-0.68(0.69)$ \\
\hline 15 mo RM baby foods & 537 & -0.52 & -0.41 & -0.64 & $-2.04(0.59)$ & $-1.96(0.59)$ & $-1.57(0.66)$ \\
\hline 15 mo Rev HM traditional & 543 & 0.13 & -0.01 & 0.51 & $-0.44(0.53)$ & $-0.59(0.57)$ & $-0.12(0.50)$ \\
\hline 24 mo HM traditional & 358 & -2.67 & -2.64 & -2.10 & $-1.44(0.54)^{3}$ & $-1.38(0.56)$ & $-1.09(0.58)$ \\
\hline 24 mo Contemporary & 240 & 4.57 & 6.46 & 0.93 & $2.08(1.25)$ & $2.60(1.49)$ & $0.88(1.32)$ \\
\hline 24 mo Discretionary & 223 & -4.58 & -5.31 & -2.77 & $-1.48(1.15)$ & $-1.73(1.15)$ & $-0.86(1.17)$ \\
\hline
\end{tabular}




\begin{tabular}{|c|c|c|c|c|c|c|c|}
\hline 24 mo Ready-to-eat foods & 374 & 2.73 & 3.35 & 0.96 & $2.27(0.56)$ & $2.26(0.50)$ & $1.63(0.61)$ \\
\hline
\end{tabular}

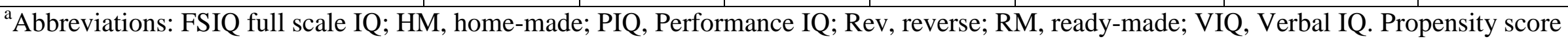
calculated as the probability of being in the highest compared with the lowest tertile of dietary pattern scores given the following covariables; sex, gestational age, birth weight, ethnicity, singleton/twin pregnancies, parity, maternal education, social class, marital status, family income, smoking, HOME questionnaire and other dietary pattern scores at current and previous times. Propensity scores matched in the high- compared with the lowest tertile by nearest neighbor method with calipers set to $0.2 * \mathrm{SD}$ [21]. IQ was measured by a short form of the Wechsler Intelligence Scale for Children version III (WISC-III).

${ }^{b}$ Values show average treatment effect and the between dataset standard deviation in parentheses for all 20 imputed datasets. Datasets were combined according to Rubin's rules [17]. The number of matches not shown as it differs for each of the 20 datasets, but is $>2000$ for all comparisons at 6 and 15 months and $>1000$ at 24 months. 


\section{FIG. 1 LEGEND \& TITLE}

TITLE: Participant data at each stage of study ${ }^{a}$

\section{Footnotes:}

${ }^{\text {a }}$ Abbreviations: ALSPAC, Avon longitudinal study of parents and children; IQ, intelligence quotient; WISC, Wechsler Intelligence Scale for Children version III

${ }^{\mathrm{b}}$ Triplets and quadruplets are excluded from all ALSPAC datasets to protect their identity

${ }^{\mathrm{c}}$ All participants who were part of the core cohort and survived to age 1 were invited to complete diet questionnaires at $6,15,24$ months and assessments of IQ at 8 years. The current study was undertaken on participants who had at least one WISC IQ subscale measured at 8 years $(n=7097)$.

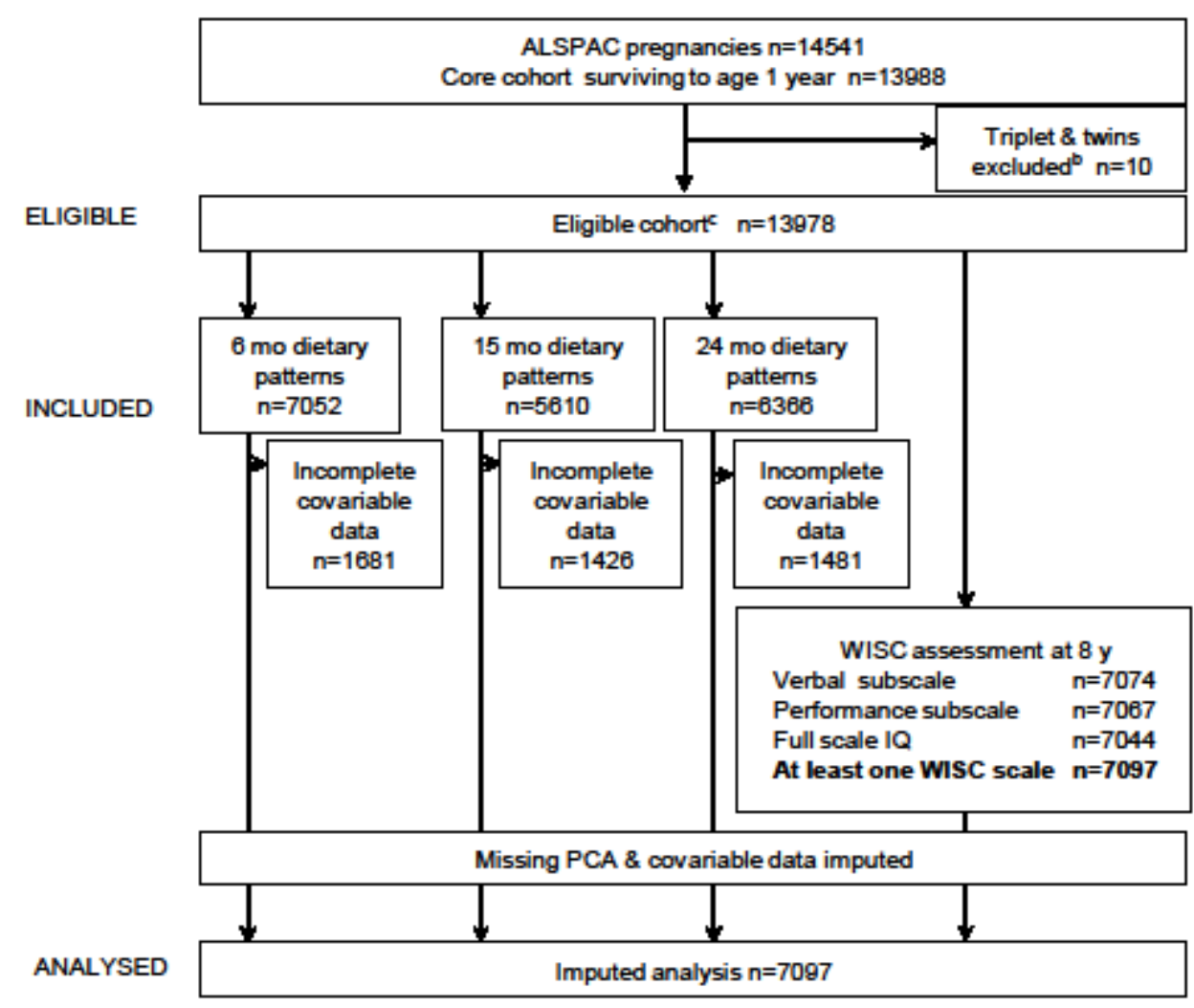

